\title{
A FOOTNOTE TO THE POINCARÉ COMPLETE REDUCIBILITY THEOREM
}

\author{
HenRIK H. Martens
}

\begin{abstract}
Poincaré's work on the reduction of abelian integrals contains implicitly an algorithm for the expression of a theta function as a sum of products of theta functions of fewer variables in the presence of reduction. The aim of this paper is to give explicit formulations and reasonably complete proofs of Poincaré's results.
\end{abstract}

\section{Introduction}

In a paper of 1935 A. A. Albert [1] gave a proof of the fact that if a Riemann matrix is on the form

$$
\left(\begin{array}{cc}
\omega_{1} & 0 \\
\omega_{3} & \omega_{2}
\end{array}\right)
$$

then it is isogenous (isomorphic in Albert's terminology) to the matrix

$$
\left(\begin{array}{cc}
\omega_{1} & 0 \\
0 & \omega_{2}
\end{array}\right)
$$

and $\omega_{1}$ and $\omega_{2}$ are Riemann matrices. He attributed the result to Poincaré, and it has since been referred to as the Poincaré Complete Reducibility Theorem. Albert's goal was the classification of multiplier algebras of Riemann matrices, and the theorem plays a key role in his decisive work on this problem.

Poincaré, however, was concerned with an entirely different problem. In a manuscript of 1874 , (which later appeared in the Acta Mathematica, [4]) S. Kowalevsky had quoted two results of Weierstrass on the

Work on this paper was carried out while the author enjoyed (sic!) the hospitality and support of the Institut Mittag-Leffler, the Mathematical 1nstitute of the University of Copenhagen, and the Centre de Recerca Matemàtica, Barcelona. 
reduction of abelian integrals to elliptic integrals, and the expression of the associated theta functions in terms of products of theta functions of fewer variables, without proof. Poincaré's goal was to provide a proof of the theorems, and of their gcneralisation to arbitrary cases of reduction. While the existence of a representation of theta functions of $n$ variables in terms of theta functions of fewer variables in the presence of reduction may be deduced from Albert's formulation, Poincarć's papers provide algorithms for the explicit computation of the representation. Since this aspect of his work doesn't appear to have bcen adequately reported in the litcrature, - his papers (see [5], [6] and volume III of his Collected Works) are rather sketchy - and since it is relevant to some of the work currently going on in the field, - e.g. that of Matveev and his coworkers, see [7] and [2, and its bibliography] - the following exposition seemed warranted.

\section{Reducible Abelian Integrals and Theta Functions}

Let $Z$ be an $n \times n$ matrix of complex numbers satisfying the Riemann relations:

$$
\begin{gathered}
{ }^{t} Z=Z \\
\Im(Z)>0,
\end{gathered}
$$

i.e. $Z$ is symmetric and the matrix of irnaginary parts of its entries is positive definite. Consider its associated theta function

$$
\theta(z ; Z)=\sum_{m} \operatorname{cxp}\left[\pi i^{l} m(Z m+2 z)\right]
$$

where the summation is over all $n$-vectors $m$ of integers, $z \in C^{n}$, and $m$ and $z$ are to be thought of as column vectors.

If the matrix $Z$ splits into a direct sum

$$
Z=\left(\begin{array}{cc}
Z_{1} & 0 \\
0 & Z_{2}
\end{array}\right)
$$

of an $n_{1} \times n_{1}$ and an $n_{2} \times n_{2}$ matrix, then, with $z={ }^{t}\left(z_{1}, z_{2}\right)$, the theta function will split into a product

$$
\theta(z ; Z)=\theta\left(z_{1} ; Z_{1}\right) \theta\left(z_{2} ; Z_{2}\right),
$$

by an obvious rearangement of the defining series. 
More generally, reduction to theta functions of lower dimension is possible when $Z$ is on the form

$$
Z=\left(\begin{array}{cc}
Z_{1} & Q \\
{ }^{t} Q & Z_{2}
\end{array}\right)
$$

whcre $Q$ is an $n_{1} \times n_{2}$ matrix of rationals, as shown by the following argument:

We have

$$
{ }^{t} m(Z n+2 z)={ }^{t} m_{1}\left(Z_{1} m_{1}+2 z_{1}+2 Q m_{2}\right)+{ }^{t} m_{2}\left(Z_{2} m_{2}+2 z_{2}\right) .
$$

Multiplying by $\pi i$, cxponentiating, and summing over $m_{1}$, we get

$$
\theta(z ; Z)=\sum_{m_{2}} \theta\left(z_{1}+Q m_{2} ; Z_{1}\right) \exp \left[\pi i^{t} m_{2}\left(Z_{2} m_{2}+2 z_{2}\right)\right] .
$$

Now, let $D=\operatorname{diag}\left(d_{1}, d_{2}, \ldots, d_{n_{2}}\right)$ be a diagonal matrix of positive integers such that $Q D$ is a matrix of integers. Writc.

$$
m_{2}=\ddot{m}_{2}+D k_{2}
$$

where $0 \leq \bar{m}_{2}^{j}<d_{j}$, and observe that

$$
\theta\left(z_{1}+Q m_{2} ; Z_{1}\right)=\theta\left(z_{1}+Q \bar{m}_{2} ; Z_{1}\right) .
$$

Fixing $\vec{m}_{2}$ we may therefore sum over $k_{2}$ to get.

(*) $\quad \theta(z ; Z)=\sum_{\bar{m}_{2}} \theta\left(z_{1}+Q \bar{m}_{2} ; Z_{1}\right) \times \theta\left[\begin{array}{c}D^{-1} \bar{m}_{2} \\ 0\end{array}\right]\left(D z_{2} ; D Z_{2} D\right)$.

where

$$
\begin{aligned}
& \theta\left[\begin{array}{c}
D^{-1} \bar{m}_{2} \\
0
\end{array}\right]\left(D z_{2 ;} D Z_{2} D\right)= \\
& \sum_{k_{2}} \exp \left[\pi i^{l}\left(k_{2}+D^{-1} \bar{m}_{2}\right)\left(D Z_{2} D\left(k_{2}+D^{-1} \bar{m}_{2}\right)+2 D z_{2}\right)\right] \\
& \quad=\sum_{k_{2}} \exp \left[\pi i^{l} m_{2}\left(Z_{2} m_{2}+2 z_{2}\right)\right]
\end{aligned}
$$

is the standard theta function with characteristics defined generally, in dimension $n$, by

$$
\theta\left[\begin{array}{c}
r \\
s
\end{array}\right](z ; Z)=\sum_{m} \exp \left[\pi i^{l}(m+r)(Z(m+r)+2(z+s))\right],
$$


where $r$ and $s$ are real $n$-vectors (see, e.g., Conforto [3].)

Suppose again that

$$
Z=\left(\begin{array}{ll}
Z_{1} & Q \\
t Q & Z_{2}
\end{array}\right)
$$

and consider the full period matrix

$$
\left(\begin{array}{cccc}
E_{1} & 0 & Z_{1} & Q \\
0 & E_{2} & { }^{t} Q & Z_{2}
\end{array}\right)
$$

where $E_{j}$ denotes an identity matrix of the appropriate size, and 0 is a (generic) matrix of zeros. We clearly have an equation

$$
\left(\begin{array}{ll}
d E_{1} & 0
\end{array}\right)\left(\begin{array}{cccc}
E_{1} & 0 & Z_{1} & Q \\
0 & E_{2} & { }^{t} Q & Z_{2}
\end{array}\right)=\left(\begin{array}{ll}
E_{1} & Z_{1}
\end{array}\right)\left(\begin{array}{cccc}
d E_{1} & 0 & 0 & d Q \\
0 & 0 & d E_{1} & 0
\end{array}\right)
$$

where $d$ is an integer chosen so that $d Q$ is a matrix of integers.

We say, in general, that an $n \times 2 n$ period matrix $\left(\begin{array}{ll}E & Z\end{array}\right)$ admits reduction if it satisfics an equation

$$
H \times\left(\begin{array}{ll}
E & Z
\end{array}\right)=\Pi \times M,
$$

where $\Pi$ is an $m \times 2 m$ matrix of complex numbers, $H$ is a maximal rank $m \times n$ matrix of complex numbers, and $M$ is a maximal rayk $2 m \times 2 n$ matrix of integers, $1 \leq m<n$. We have thus shown that a period matrix of the form considered above necessarily admits reduction.

Poincarć showed, conversely, that if a period matrix $\left(\begin{array}{ll}E & Z\end{array}\right)$ admits reduction, then it is symplectically equivalent to a matrix on the form indicated.

(Recall that a $2 n \times 2 n$ matrix $T$ of integers is said to be symplectic if $T J^{t} T=J$ where

$$
J=\left(\begin{array}{cc}
0 & E \\
-E & 0
\end{array}\right)
$$

is the canonical intersection matrix.)

Theorem (Weierstrass - Poincaré). Let $\left(\begin{array}{ll}E & Z\end{array}\right)$ be an $n \times 2 n$ matrix satisfying the Ricmann conditions and admiting reduction

$$
H \times\left(\begin{array}{ll}
E & Z
\end{array}\right)=\Pi \times M
$$

where $\Pi$ is an $m \times 2 m$ matrix of complex numbers, $1 \leq m<n$. 
Then there is an $n \times n$ non-singular matrix $A$ of complex numbers, and a $2 n \times 2 n$ symplectic unimodular matrix $T$ such that

$$
\left(\begin{array}{ll}
E & Z
\end{array}\right) \times T=A \times\left(\begin{array}{cccc}
E_{1} & 0 & Z_{1} & Q \\
0 & E_{2} & { }^{t} Q & Z_{2}
\end{array}\right),
$$

where $Z_{1}$ and $Z_{2}$ are $m \times m$ and $(n-m) \times(n-m)$ matrices satisfying the Riemann relations, and $Q$ is an $m \times(n-m)$ matrix of rational numbers whose non-zero entries, if any, are confined to on initial string along the main diagonal, $q_{j j}$.

Poincaré's proof is based on the existence of a normal form for $M$, which may be of independent interest.

Normal Form Lemma. Let $1 \leq m<n$, and let $M$ be $a 2 m \times 2 n$ matrix of maximal rank with integer entries such that $M J^{t} M$ is nonsingular. Then

$$
M=S N T
$$

where $S$ is a $2 m \times 2 m$ non-singular matrix of integers, $T$ is a $2 n \times 2 n$ symplectic unimodular matrix, and $N$ is a $2 n \times 2 n$ matrix of integers on block form

$$
N=\left(\begin{array}{cccc}
E_{1} & 0 & 0 & 0 \\
0 & X & \Delta & 0
\end{array}\right)
$$

where $E_{1}$ is an $m \times m$ identity matrix, $\Delta$ is a diagonal matrix of integers each of which is a multiple of the following, and $X$ is an $m \times(n-m)$ matrix where $x_{j j}=1$ for all $j \leq r$ for some $r$ with $0 \leq r \leq n-n$, and the remaining entries are zero.

Given the lemma, the proof of the theorem is straightforward. If a matrix $M$ occurs in a reduction equation

$$
H \times\left(\begin{array}{ll}
E & Z
\end{array}\right)=\Pi \times M,
$$

then $M J^{i} M$ must be non-singular.

To see this, observe that by an easy calculation the Riemann conditions for $Z$ are equivalent to the equations

$$
\left(\begin{array}{ll}
E & Z
\end{array}\right) J^{t}\left(\begin{array}{ll}
E & Z
\end{array}\right)=0
$$

and

$$
i\left(\begin{array}{ll}
E & Z
\end{array}\right) J^{l}\left(\begin{array}{ll}
E & \bar{Z}
\end{array}\right)>0 .
$$


It follows that $\Pi M J^{t} M^{t} \Pi=0$ and that $i \Pi M J^{t} M^{t} \bar{\Pi}$ is positive definite, since $H$ is of maximal rank. Since

$$
\left(\frac{\Pi}{\bar{\Pi}}\right)\left(M T^{t} M\right)^{t}\left(\frac{\Pi}{\bar{\Pi}}\right)=\left(\begin{array}{cc}
0 & \Pi M J^{t} M^{t} \bar{\Pi} \\
\left.\bar{\Pi} M J^{t} M^{t} \Pi\right) & 0
\end{array}\right),
$$

it follows that the factors on the left are nonsingular.

Now, from the reduction equation we get, by the lemma,

$$
H \times\left(\begin{array}{ll}
E & Z
\end{array}\right)=\Pi \times S \times\left(\begin{array}{cccc}
E_{1} & 0 & 0 & 0 \\
0 & X & \Delta & 0
\end{array}\right) \times T .
$$

Writing $\Pi \times S$ as $\left(\Pi_{1} \quad \Pi_{2}\right)$, and multiplying out, we have

$$
\begin{aligned}
H \times\left(\begin{array}{ll}
E & Z
\end{array}\right) & =\left(\begin{array}{llll}
\Pi_{1} & \Pi_{2} X & \Pi_{2} \Delta & 0
\end{array}\right) \times T \\
& =\left(\begin{array}{llll}
\Pi_{2} \Delta & 0 & -\Pi_{1} & -\Pi_{2} X
\end{array}\right) \times J T .
\end{aligned}
$$

Since the matrix $J T$ is symplectic unimodular,

$$
\left(\begin{array}{ll}
E & Z
\end{array}\right)(J T)^{-1}=G \times\left(\begin{array}{ll}
E & Z^{\prime}
\end{array}\right),
$$

where $Z^{\prime}$ is an $n \times n$ matrix satisfying the Riemann relations and $G$ is a non-singular $n \times n$ matrix. (Write $\left(\begin{array}{ll}E & Z\end{array}\right)(J T)^{-1}=\left(\begin{array}{ll}\Omega_{1} & \Omega_{2}\end{array}\right)=\Omega$, show that, $i \Omega J^{t} \bar{\Omega}$ is positive definite and conclude that $\Omega_{1}$ and $\Omega_{2}$ must be non-singular.) Then

$$
H G\left(\begin{array}{ll}
E & Z^{\prime}
\end{array}\right)=\left(\begin{array}{llll}
\Pi_{2} \Delta & 0 & -\Pi_{1} & -\Pi_{2} X
\end{array}\right)
$$

wherce it follows that

$$
H G=\left(\begin{array}{ll}
\Pi_{2} \Delta & 0
\end{array}\right) .
$$

Since $H$ was assumed of maximal rank, $\Pi_{2} \Delta$ must be non-singular. Then

$$
\left(\begin{array}{ll}
E & Z^{\prime}
\end{array}\right)=\left(\begin{array}{cccc}
E_{1} & 0 & -\Delta^{-1} \Pi_{2}^{-1} \Pi_{1} & -\Delta^{-1} X \\
0 & E_{2} & -{ }^{t} X \Delta^{-1} & Z_{2}
\end{array}\right),
$$

with some $(n-m) \times(n-m)$ matrix $Z_{2}$. Since this is on the postulated form, the proof of the theorem is completed.

Note that the matrix $Z^{\prime}$ may be computed from $Z$ once the symplectic matrix $T$ of the lemma has been detcrmined. The remainder of the paper is devoted to a proof of the lemma which, in fact, will give an algorithm to determine $T$, given $M$. Thus the proof of the theorem is constructive.

Bounds on the number of terms in the representation (*) may be obtained from the entries of the matrix $\Delta$ and the non zero entries of $X$. When the reduction arises as a consequence of mappings of Riemann surfaces, the entries of $\Delta$ will be bounded by the degree of the map.

If all entries of the matrix $X$ are zero, the matrix $Z^{\prime}$ will split into a dircet surn. This cannot happen when $\left(\begin{array}{lll}E & Z\end{array}\right)$ is the canonical period matrix of a closed Ricmann surface, and thus excludes certain matrices $M$ from represcnting induced homology maps of surfaces. 


\section{Poincaré Normal Form for $2 \times 2 n$ Matrices}

It will be instructive first to consider the special case of a $2 \times 2 n$ matrix, $M$, of integers. In this casc, $M J^{t} M$ is a $2 \times 2$ skew-symmetric matrix of integers, and hence may be assumed to be on the form

$$
d\left(\begin{array}{cc}
0 & 1 \\
-1 & 0
\end{array}\right)
$$

where $d$ is a non-vanishing integer.

We review some clementary changes that may be effected by multiplying $M$ on the right with a symplectic matrix $T$. For this purpose it is convenient to think of the matrix as made up of two $2 \times n$ blocks, $M=\left(\begin{array}{ll}M_{1} & M_{2}\end{array}\right)$.

It is easily verificd that the following $2 n \times 2 n$ matrices are symplectic:

$$
\left(\begin{array}{ll}
E & S \\
0 & E
\end{array}\right)
$$

$$
\left(\begin{array}{ll}
E & 0 \\
S & E
\end{array}\right)
$$

where $E$ is the $n \times n$ identity matrix, and $S$ is a symmetric $n \times n$ matrix of integers, and

$$
\left(\begin{array}{cc}
A & 0 \\
0 &
\end{array}\right.
$$

where $A$ is a unimodular matrix (of integers.)

With these matrices, the following sirnple changes may be effected:

A. We may add a multiple of a column of one block to the corresponding column in the other biock, by using a symplectic matrix of the form (1) or (2) with a diagonal $S$. In particular, we may intcrchange two corresponding columns, provided we change the sign of one of them.

B. We may add a multiple of any column of a block to any other column of the same block, using a symplectic matrix of the form (3), provided we subtract corresponding columns in the other block in the opposite order.

C. We may permute the columns of a block, asing a symplectic matrix of the form (3), provided the columns of the other block are permuted accordingly. 
Note that, in the simple case of a $2 \times 2 n$ matrix, adding a multiple of one row to the other, or interchanging rows and changing the sign of one of them, are effected by multiplication on the left by a symplectic $2 \times 2$ matrix. In the general case, row operations may be carried out corresponding to the operations outlined above, viewing the matrix as consisting of two $m \times 2 n$ blocks.

Using operation (A) on two corresponding columns from the two blocks, we can always arrange that the first entry in the column of the second block is zero. Hence we get a matrix on the form

$$
\left(\begin{array}{llllllll}
\star & \star & \cdots & \star & 0 & 0 & \cdots & 0 \\
\star & \star & \cdots & \star & \star & \star & \cdots & \star
\end{array}\right),
$$

where the $\star$ denotes an unknown entry. Using operations (B) and (C), we may next reduce the first block to the form

$$
\left(\begin{array}{cccccccc}
r & 0 & \cdots & 0 & 0 & 0 & \cdots & 0 \\
\star & \star & \cdots & \star & \star & \star & \cdots & \star
\end{array}\right),
$$

where $r$ is a non-zero integer. We note that the compensating operations in the second block do not affect the zeros in the first line.

Disregarding the first column of each block, we may now repeat the procedure on the remaining columns. This will not affect the zeros in the first row, and will produce a matrix of the form

$$
\left(\begin{array}{cccccccccc}
r & 0 & 0 & \cdots & 0 & 0 & 0 & 0 & \cdots & 0 \\
x & y & 0 & \cdots & 0 & s & 0 & 0 & \cdots & 0
\end{array}\right)
$$

where $r s=d$.

We may further assume that $r$ divides $x, y$ and $s$, since otherwise we could subtract a suitable multiple of the first row from the second, interchange rows, repeat the procedure $a b$ initio, and arrive at a matrix of the same form with a smaller value for $r$. Hence we may subtract a multiple the first row from the second, and get a matrix on the form

$$
r \times\left(\begin{array}{cccccccccc}
1 & 0 & 0 & \cdots & 0 & 0 & 0 & 0 & \cdots & 0 \\
0 & a & 0 & \cdots & 0 & b & 0 & 0 & \cdots & 0
\end{array}\right) .
$$

Assuming $a \neq 0$, the following trick of Picard cnables us to replace $a$ by the greatest common divisor of $a$ and $b$, multiplying the matrix on the right by symplectic matrices:

Consider a $2 \times 4$ matrix

$$
\left(\begin{array}{llll}
1 & 0 & 0 & 0 \\
0 & a & b & 0
\end{array}\right)
$$


Use the symplectic matrix

$$
\left(\begin{array}{llll}
1 & 0 & 0 & 0 \\
0 & 1 & 0 & 1 \\
0 & 0 & 1 & 0 \\
0 & 0 & 0 & 1
\end{array}\right)
$$

to produce

$$
\left(\begin{array}{llll}
1 & 0 & 0 & 0 \\
0 & a & b & a
\end{array}\right)
$$

Then use the symplectic matrix

$$
\left(\begin{array}{llll}
1 & 0 & 0 & 0 \\
0 & 1 & 0 & 0 \\
0 & \beta & 1 & 0 \\
\beta & \alpha & 0 & 1
\end{array}\right)
$$

to produce

$$
\left(\begin{array}{cccc}
1 & 0 & 0 & 0 \\
\beta a & c & b & a
\end{array}\right)
$$

where $c=(1+\alpha) a+\beta b=\operatorname{gcd}(a, b)$. Finally, use the symplectic matrix

$$
\left(\begin{array}{cccc}
1 & 0 & 0 & 0 \\
0 & 1 & 0 & -a / c \\
0 & 0 & 1 & 0 \\
0 & 0 & 0 & 1
\end{array}\right)
$$

to produce

$$
\left(\begin{array}{cccc}
1 & 0 & 0 & 0 \\
\beta a & c & b & 0
\end{array}\right)
$$

and multiply on the left, with the symplectic matrix

$$
\left(\begin{array}{cc}
1 & 0 \\
-\beta a & 1
\end{array}\right)
$$

to produce

$$
\left(\begin{array}{llll}
1 & 0 & 0 & 0 \\
0 & c & b & 0
\end{array}\right)
$$


The trick can evidently be used, mutatis mutandis, on any two pairs of corresponding columns from the two blocks, and thus reduces our matrix above to the form

$$
r \times\left(\begin{array}{cccccccccc}
1 & 0 & 0 & \cdots & 0 & 0 & 0 & 0 & \cdots & 0 \\
0 & c & 0 & \cdots & 0 & b & 0 & 0 & \cdots & 0
\end{array}\right),
$$

obtained from our original matrix by multiplying on the right and left, if necessary, by symplectic matrices. We may formulate the result of this section:

Lemma. Let $M$ be a $2 \times 2 n$ matrix of integers of maximal rank satisfying

$$
M J^{t} M=d\left(\begin{array}{cc}
0 & 1 \\
-1 & 0
\end{array}\right)
$$

Then

$$
M=S N T,
$$

where $S$ and $T$ are symplectic unimodular matrices, and

$$
N=r \times\left(\begin{array}{cccccccccc}
1 & 0 & 0 & \cdots & 0 & 0 & 0 & 0 & \cdots & 0 \\
0 & s & 0 & \cdots & 0 & t & 0 & 0 & \cdots & 0
\end{array}\right)
$$

where $r^{2} t=d$ and $s$ either vanishes or is a divisor of $t$.

\section{Poincaré Normal Form - General Case}

We now turn to the proof of the Normal Form Lemma. We shall represcnt $M$ by an array

$$
\left(\begin{array}{cccccccccccccccc}
\star & \star & \star & \cdots & \star & \star & \cdots & \star & \star & \star & \star & \cdots & \star & \star & \cdots & \star \\
\star & \star & \star & \cdots & \star & \star & \cdots & \star & \star & \star & \star & \cdots & \star & \star & \cdots & \star \\
\star & \star & \star & \cdots & \star & \star & \cdots & \star & \star & \star & \star & \cdots & \star & \star & \cdots & \star \\
\vdots & \vdots & \vdots & \ddots & \vdots & \vdots & & \vdots & \vdots & \vdots & \vdots & \ddots & \vdots & \vdots & & \vdots \\
\star & \star & \star & \cdots & \star & \star & \cdots & \star & \star & \star & \star & \cdots & \star & \star & \cdots & \star \\
& & & & & & & & & & & & & & & \\
\star & \star & \star & \cdots & \star & \star & \cdots & \star & \star & \star & \star & \cdots & \star & \star & \cdots & \star \\
\star & \star & \star & \cdots & \star & \star & \cdots & \star & \star & \star & \star & \cdots & \star & \star & \cdots & \star \\
\star & \star & \star & \cdots & \star & \star & \cdots & \star & \star & \star & \star & \cdots & \star & \star & \cdots & \star \\
\vdots & \vdots & \vdots & \ddots & \vdots & \vdots & & \vdots & \vdots & \vdots & \vdots & \ddots & \vdots & \vdots & & \vdots \\
\star & \star & \star & \cdots & \star & \star & \cdots & \star & \star & \star & \star & \cdots & \star & \star & \cdots & \star
\end{array}\right),
$$

where the stars donote arbitrary cntries to be specified as wc proceed. The argument will be broken up in a series of steps. 
Step 1. By factoring out a lower triangular matrix of integers on the left, if necessary, we may assume that the greatest common divisor of the $2 m \times 2 m$ subdetcrminants of $M$ is 1 . Observe that this property is preserved under multiplication on the right or the left by a unimodular matrix. (Multiplication on the left does not alter the values of the subdetcrminants. Multiplication on the right produces subdeterminants whose columns are linear combinations of those of the original ones. The new determinants will thercforc be linear combinations of the original ones, and the greatest common divisor will not decrease. By unimodularity, we may get back to the original matrix by the same kind of operation, hence the greatest common divisor cannot increase either.)

Step 2. By a result of Frobenius (scc [3]), factoring out a unimodular matrix on the left, if necessury, we may assume that

$$
M \cdot J^{t} M=\left(\begin{array}{cc}
0 & \Delta \\
-\Delta & 0
\end{array}\right)
$$

whore $\Delta$ is a diagonal matrix of integers each of which is a multiple of the next. Observe that this property is preserved under multiplication of the matrix on the right by a symplectic matrix.

Step 3. Performing clcmentary operations on the columns as in the case of a $2 \times 2 \pi$ matrix, we can bring the matrix on the form:

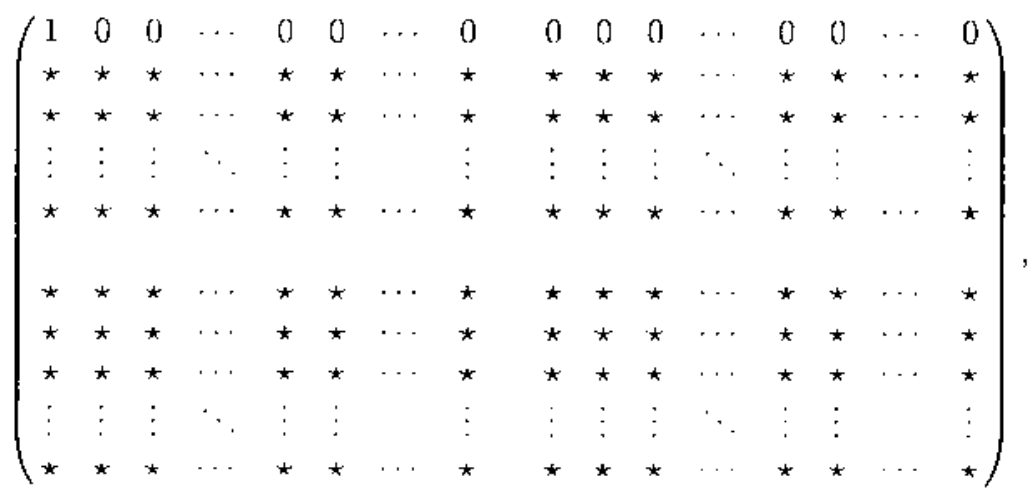

where the value 1 in the first row is a consequence of the assumption in Step 1 , and the assumption of maximal rank. Writing $M$ as $\left(\begin{array}{ll}M_{1} & M_{2} \\ M_{3} & M_{4}\end{array}\right)$, we have

$$
M J^{t} M=\left(\begin{array}{cc}
-M_{2}{ }^{t} M_{1}+M_{1}{ }^{t} M_{2} & -M_{2}{ }^{t} M_{3}+M_{1}{ }^{t} M_{4} \\
-M_{1}{ }^{t} M_{1}+M_{3}{ }^{t} M_{2} & \cdots M_{4}{ }^{t} M_{3}+M_{3}{ }^{t} M_{4}
\end{array}\right)
$$


and conclude from the assumption of Stcp 2 that $M_{1}{ }^{l} M_{2}$ is a symmetric matrix. This forces the first column of $M_{2}$ to be zero, and we have reduced $M$ to the form

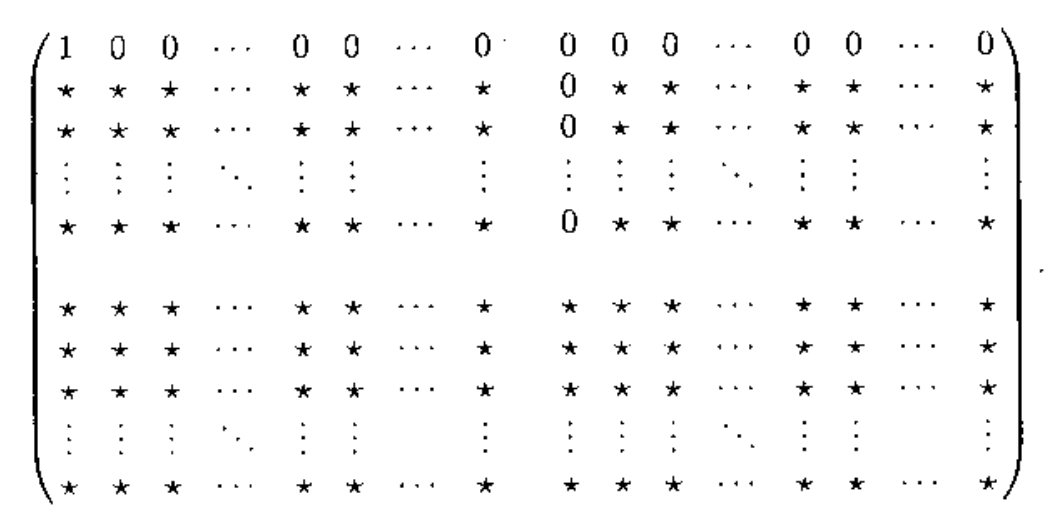

Disregarding the first column of each block, and repeating the operation on the remaining columns, we get the form

$$
\left(\begin{array}{cccccccccccccccc}
\star & 0 & 0 & \cdots & 0 & 0 & \cdots & 0 & 0 & 0 & 0 & \cdots & 0 & 0 & \cdots & 0 \\
\star & 1 & 0 & \cdots & 0 & 0 & \cdots & 0 & 0 & 0 & 0 & \cdots & 0 & 0 & \cdots & 0 \\
\star & \star & \star & \cdots & \star & \star & \cdots & \star & 0 & 0 & \star & \cdots & \star & \star & \cdots & \star \\
\vdots & \vdots & \vdots & \ddots & \vdots & \vdots & & \vdots & \vdots & \vdots & \vdots & \ddots & \vdots & \vdots & & \vdots \\
\star & \star & \star & \cdots & \star & \star & \cdots & \star & 0 & 0 & \star & \cdots & \star & \star & \cdots & \star \\
\star & & & & & & & & & & & & & & & \\
\star & \star & \star & \cdots & \star & \star & \cdots & \star & \star & \star & \star & \cdots & \star & \star & \cdots & \star \\
\star & \star & \star & \cdots & \star & \star & \cdots & \star & \star & \star & \star & \cdots & \star & \star & \cdots & \star \\
\vdots & \vdots & \vdots & \ddots & \vdots & \vdots & & \vdots & \vdots & \vdots & \vdots & \ddots & \vdots & \vdots & & \vdots \\
\star & \star & \star & \cdots & \star & \star & \cdots & \star & \star & \star & \star & \cdots & \star & \star & \cdots & \star
\end{array}\right),
$$

where the 1 on the diagonal again is a consequence of the assumption in Step 1, and the maximal rank. This process may obviusly be continued 
until we reach the form

$$
\left(\begin{array}{cccccccccccccccc}
1 & 0 & 0 & \cdots & 0 & 0 & \cdots & 0 & 0 & 0 & 0 & \cdots & 0 & 0 & \cdots & 0 \\
\star & 1 & 0 & \cdots & 0 & 0 & \cdots & 0 & 0 & 0 & 0 & \cdots & 0 & 0 & \cdots & 0 \\
\star & \star & 1 & \cdots & 0 & 0 & \cdots & 0 & 0 & 0 & 0 & \cdots & 0 & 0 & \cdots & 0 \\
\vdots & \vdots & \vdots & \ddots & \vdots & \vdots & & \vdots & \vdots & \vdots & \vdots & \ddots & \vdots & \vdots & & \vdots \\
\star & \star & \star & \cdots & 1 & 0 & \cdots & 0 & 0 & 0 & 0 & \cdots & 0 & 0 & \cdots & 0 \\
\star & \star & \star & \cdots & \star & \star & \cdots & \star & \star & \star & \star & \cdots & \star & \star & \cdots & \star \\
\star & \star & \star & \cdots & \star & \star & \cdots & \star & \star & \star & \star & \cdots & \star & \star & \cdots & \star \\
\star & \star & \star & \cdots & \star & \star & \cdots & \star & \star & \star & \star & \cdots & \star & \star & \cdots & \star \\
\vdots & \vdots & \vdots & \ddots & \vdots & \vdots & & \vdots & \vdots & \vdots & \vdots & \ddots & \vdots & \vdots & & \vdots \\
\star & \star & \star & \cdots & \star & \star & \cdots & \star & \star & \star & \star & \cdots & \star & \star & \cdots & \star
\end{array}\right),
$$

and multiplication on the right by a symplectic matrix of the form

$$
\left(\begin{array}{cc}
A & 0 \\
0 & { }^{\iota} A^{-1}
\end{array}\right)
$$

may be used to obtain the form

$$
\left(\begin{array}{cccccccccccccccc}
1 & 0 & 0 & \cdots & 0 & 0 & \cdots & 0 & 0 & 0 & 0 & \cdots & 0 & 0 & \cdots & 0 \\
0 & 1 & 0 & \cdots & 0 & 0 & \cdots & 0 & 0 & 0 & 0 & \cdots & 0 & 0 & \cdots & 0 \\
0 & 0 & 1 & \cdots & 0 & 0 & \cdots & 0 & 0 & 0 & 0 & \cdots & 0 & 0 & \cdots & 0 \\
\vdots & \vdots & \vdots & \ddots & \vdots & \vdots & & \vdots & \vdots & \vdots & \vdots & \ddots & \vdots & \vdots & & \vdots \\
0 & 0 & 0 & \cdots & 1 & 0 & \cdots & 0 & 0 & 0 & 0 & \cdots & 0 & 0 & \cdots & 0 \\
\star & \star & \star & \cdots & \star & \star & \cdots & \star & \star & \star & \star & \cdots & \star & \star & \cdots & \star \\
\star & \star & \star & \cdots & \star & \star & \cdots & \star & \star & \star & \star & \cdots & \star & \star & \cdots & \star \\
\star & \star & \star & \cdots & \star & \star & \cdots & \star & \star & \star & \star & \cdots & \star & \star & \cdots & \star \\
\vdots & \vdots & \vdots & \ddots & \vdots & \vdots & & \vdots & \vdots & \vdots & \vdots & \ddots & \vdots & \vdots & & \vdots \\
\star & \star & \star & \cdots & \star & \star & \cdots & \star & \star & \star & \star & \cdots & \star & \star & \cdots & \star
\end{array}\right) .
$$

The formula

$$
M J^{t} M=\left(\begin{array}{cc}
-M_{2}{ }^{t} M_{1}+M_{1}{ }^{t} M_{2} & -M_{2}{ }^{t} M_{3}+M_{1}{ }^{t} M_{4} \\
-M_{4}{ }^{t} M_{1}+M_{3}{ }^{t} M_{2} & -M_{4}{ }^{t} M_{3}+M_{3}{ }^{t} M_{4}
\end{array}\right),
$$

now enables us to conclude that $M_{1}^{t} M_{4}=\Delta$, and so $M_{4}$ must have $\Delta$ 
as an initial $m \times m$ block. Hence the matrix has the form

$$
\left(\begin{array}{cccccccccccccccc}
1 & 0 & 0 & \cdots & 0 & 0 & \cdots & 0 & 0 & 0 & 0 & \cdots & 0 & 0 & \cdots & 0 \\
0 & 1 & 0 & \cdots & 0 & 0 & \cdots & 0 & 0 & 0 & 0 & \cdots & 0 & 0 & \cdots & 0 \\
0 & 0 & 1 & \cdots & 0 & 0 & \cdots & 0 & 0 & 0 & 0 & \cdots & 0 & 0 & \cdots & 0 \\
\vdots & \vdots & \vdots & \ddots & \vdots & \vdots & & \vdots & \vdots & \vdots & \vdots & \ddots & \vdots & \vdots & & \vdots \\
0 & 0 & 0 & \cdots & 1 & 0 & \cdots & 0 & 0 & 0 & 0 & \cdots & 0 & 0 & \cdots & 0 \\
& & & & & & & & & & & & & & & \\
\star & \star & \star & \cdots & \star & \star & \cdots & \star & d_{1} & 0 & 0 & \cdots & 0 & \star & \cdots & \star \\
\star & \star & \star & \cdots & \star & \star & \cdots & \star & 0 & d_{2} & 0 & \cdots & 0 & \star & \cdots & \star \\
\star & \star & \star & \cdots & \star & \star & \cdots & \star & 0 & 0 & d_{3} & \cdots & 0 & \star & \cdots & \star \\
\vdots & \vdots & \vdots & \ddots & \vdots & \vdots & & \vdots & \vdots & \vdots & \vdots & \ddots & \vdots & \vdots & & \vdots \\
\star & \star & \star & \cdots & \star & \star & \cdots & \star & 0 & 0 & 0 & \cdots & d_{m} & \star & \cdots & \star
\end{array}\right),
$$

where each $d_{i}$ is a multiple of $d_{i+1}$.

Step 4. Operating on the last $n-m$ columns of each block, we may proceed as in the first part of Step 3 to obtain a matrix of the form

$$
\left(\begin{array}{cccccccccccccccc}
1 & 0 & \cdots & 0 & 0 & 0 & \cdots & 0 & 0 & 0 & \cdots & 0 & 0 & 0 & \cdots & 0 \\
0 & 1 & \cdots & 0 & 0 & 0 & \cdots & 0 & 0 & 0 & \cdots & 0 & 0 & 0 & \cdots & 0 \\
\vdots & \vdots & \ddots & \vdots & \vdots & & & \vdots & \vdots & \vdots & \ddots & \vdots & \vdots & & & \vdots \\
0 & 0 & \cdots & 1 & 0 & 0 & \cdots & 0 & 0 & 0 & \cdots & 0 & 0 & 0 & \cdots & 0 \\
\star & \star & \cdots & \star & k & 0 & \cdots & 0 & d_{1} & 0 & \cdots & 0 & 0 & 0 & \cdots & 0 \\
\star & \star & \cdots & \star & \star & \star & \cdots & \star & 0 & d_{2} & \cdots & 0 & \star & \star & \cdots & \star \\
\vdots & \vdots & \ddots & \vdots & \vdots & & & \vdots & \vdots & \vdots & \ddots & \vdots & \vdots & & & \vdots \\
\star & \star & \cdots & \star & \star & \star & \cdots & \star & 0 & 0 & \cdots & d_{r n} & \star & \star & \cdots & \star
\end{array}\right),
$$

where the element $k$ need not be 1 . If $k=0$, then $d_{\mathrm{I}}=1$ by the assumption of Step 1, and hence $\Delta=E$ by the assumption of Step 2 . Hence, all the starred entries of block $M_{1}$ nay be removed by subtracting appropriate multiples of the first $m$ columns. To make the operation symplectic, this rnust be compensated for by adding multiples in the opposite order of corresponding columns in the first half of the matrix, but this will not affect any of the non-starrcd entries there. We shall return to this case later.

If $k \neq 0$, it follows from the assumption in Step 1 that $k$ and $d_{1}$ must be relatively prime. Then, if $k \neq 1$, we may apply the Picard trick to colurnins 1 and $m+1$ of each block to replace $k$ by 1 . This will not alter the matrix except possibly in the starred section of $M_{3}$ and $M_{1}$, and will not violate the assumption of Step 2. From the equation

$$
M J^{t} M=\left(\begin{array}{cc}
-M_{2}{ }^{t} M_{1}+M_{1}^{t} M_{2} & -M_{2}{ }^{t} M_{3}+M_{1}^{t} M_{1} \\
-M_{1}{ }^{t} M_{1}+M_{3}{ }^{t} M_{2} & -M_{1}{ }^{t} M_{3}+M_{3}{ }^{t} M_{4}
\end{array}\right),
$$


it now follows that $M_{4}{ }^{t} M_{3}$ is symmetric. Writing out the matrices, we have

$$
M_{4}{ }^{l} M_{3}=\left(\begin{array}{cccccccc}
d_{1} & 0 & \cdots & 0 & 0 & 0 & \cdots & 0 \\
0 & d_{2} & \cdots & 0 & x_{2} & \star & \cdots & \star \\
\vdots & \vdots & \ddots & \vdots & \vdots & \vdots & & \vdots \\
0 & 0 & \cdots & d_{m} & x_{m} & \star & \cdots & \star
\end{array}\right)\left(\begin{array}{cccc}
\star & a_{2} & \cdots & a_{m} \\
b_{2} & \star & \cdots & \star \\
\vdots & \vdots & \ddots & \vdots \\
b_{m} & \star & \cdots & \star \\
1 & \star & \cdots & \star \\
0 & \star & \cdots & \star \\
\vdots & \vdots & & \vdots \\
0 & \star & \cdots & \star
\end{array}\right) \text {, }
$$

where certain elements have bcen identifed in order to facilitate the following argurnent. Comparing the first row and the first column of the product, symmetry implies $d_{1} a_{j}=d_{j} b_{j}+x_{j}$, whence it follows that $d_{j}$ is a divisor of $x_{j}$. Hence we may replace $x_{j}$ by 0 by subtracting a multiple of the column containing $d_{j}$ from the column containing $x_{j}$, as in the case above. We have then reached a matrix of the form

$$
\left(\begin{array}{cccccccccccccccc}
1 & 0 & \cdots & 0 & 0 & 0 & \cdots & 0 & 0 & 0 & \cdots & 0 & 0 & 0 & \cdots & 0 \\
0 & 1 & \cdots & 0 & 0 & 0 & \cdots & 0 & 0 & 0 & \cdots & 0 & 0 & 0 & \cdots & 0 \\
\vdots & \vdots & \ddots & \vdots & \vdots & \vdots & & \vdots & \vdots & \vdots & \ddots & \vdots & \vdots & \vdots & & \vdots \\
0 & 0 & \cdots & 1 & 0 & 0 & \cdots & 0 & 0 & 0 & \cdots & 0 & 0 & 0 & \cdots & 0 \\
& & & & & & & & & & & & & & & \\
\star & \star & \cdots & \star & 1 & 0 & \cdots & 0 & d_{1} & 0 & \cdots & 0 & 0 & 0 & \cdots & 0 \\
\star & \star & \cdots & \star & \star & \star & \cdots & \star & 0 & d_{2} & \cdots & 0 & 0 & \star & \cdots & \star \\
\vdots & \vdots & \ddots & \vdots & \vdots & \vdots & & \vdots & \vdots & \vdots & \ddots & \vdots & \vdots & \vdots & & \vdots \\
\star & \star & \cdots & \star & \star & \star & \cdots & \star & 0 & 0 & \cdots & d_{n} & 0 & \star & \cdots & \star
\end{array}\right) .
$$

The procedure of Step 4 may now be applied to the last $n-m-1$ columns of each block, yiclding at first the matrices

$$
\begin{aligned}
M_{3}= & \left(\begin{array}{cccccccccc}
\star & \star & \star & \cdots & \star & 1 & 0 & 0 & \cdots & 0 \\
\star & \star & \star & \cdots & \star & \star & k & 0 & \cdots & 0 \\
\star & \star & \star & \cdots & \star & \star & \star & \star & \cdots & \star \\
\vdots & \vdots & \vdots & \ddots & \vdots & \vdots & \vdots & \vdots & & \vdots \\
\star & \star & \star & \cdots & \star & \star & \star & \star & \cdots & \star
\end{array}\right), \\
M_{4}= & \left(\begin{array}{cccccccccc}
d_{1} & 0 & 0 & \cdots & 0 & 0 & 0 & 0 & \cdots & 0 \\
0 & d_{2} & 0 & \cdots & 0 & 0 & 0 & 0 & \cdots & 0 \\
0 & 0 & d_{3} & \cdots & 0 & 0 & \star & \star & \cdots & \star \\
\vdots & \vdots & \vdots & \ddots & \vdots & \vdots & \vdots & \vdots & & \vdots \\
0 & 0 & 0 & \cdots & d_{r n} & 0 & \star & \star & \cdots & \star
\end{array}\right)
\end{aligned}
$$


Where iggain $k$ may be zero, in which case $d_{j}=1$ for $j \geq 2$, and the starred entries in $M_{4}$ may be removed as before. If $k \neq 0$, it must be relatively prime to $d_{2}$, and we proceed as above to bring the matrices to the form

$$
M_{3}=\left(\begin{array}{cccccccccc}
\star & \star & \star & \cdots & \star & 1 & 0 & 0 & \cdots & 0 \\
\star & \star & \star & \cdots & \star & \star & 1 & 0 & \cdots & 0 \\
\star & \star & \star & \cdots & \star & \star & \star & \star & \cdots & \star \\
\vdots & \vdots & \vdots & \ddots & \vdots & \vdots & \vdots & \vdots & & \vdots \\
\star & \star & \star & \cdots & \star & \star & \star & \star & \cdots & \star
\end{array}\right),
$$

and

$$
M_{h}=\left(\begin{array}{cccccccccc}
d_{1} & 0 & 0 & \cdots & 0 & 0 & 0 & 0 & \cdots & 0 \\
0 & d_{2} & 0 & \cdots & 0 & 0 & 0 & 0 & \cdots & 0 \\
0 & 0 & d_{3} & \cdots & 0 & 0 & 0 & \star & \cdots & \star \\
\vdots & \vdots & \vdots & \ddots & \vdots & \vdots & \vdots & \vdots & & \vdots \\
0 & 0 & 0 & \cdots & d_{m} & 0 & 0 & \star & \cdots & \star
\end{array}\right)
$$

Subtracting a suitable multiple of column $m+2$ from column $m+1$ we get

$$
M_{3}=\left(\begin{array}{cccccccccc}
\star & \star & \star & \cdots & \star & 1 & 0 & 0 & \cdots & 0 \\
\star & \star & \star & \cdots & \star & 0 & 1 & 0 & \cdots & 0 \\
\star & \star & \star & \cdots & \star & \star & \star & \star & \cdots & \star \\
\vdots & \vdots & \vdots & \ddots & \vdots & \vdots & \vdots & \vdots & & \vdots \\
\star & \star & \star & \cdots & \star & \star & \star & \star & \cdots & \star
\end{array}\right) .
$$

This will not alter any unstarred entries in the matrix, as the compensating addition of a multiple of column $n+m+1$ to column $n+m+2$ only involves zero entries.

Contining these operations will, in any case, lead to a matrix where all the starred entriks in $M_{1}$ are replaced by zeros. For $M_{3}$ there are a number of possibilities.

Step 5. Consider first the case where $k=0$ in the first application of Step 4. As argued above, this implies that $d_{j}=1$ for all $j$, and the matrix is then on the form 


$$
\left(\begin{array}{cccccccccccccccc}
1 & 0 & \cdots & 0 & 0 & 0 & \cdots & 0 & 0 & 0 & \cdots & 0 & 0 & 0 & \cdots & 0 \\
0 & 1 & \cdots & 0 & 0 & 0 & \cdots & 0 & 0 & 0 & \cdots & 0 & 0 & 0 & \cdots & 0 \\
\vdots & \vdots & \ddots & \vdots & \vdots & \vdots & & \vdots & \vdots & \vdots & \ddots & \vdots & \vdots & \vdots & & \vdots \\
0 & 0 & \cdots & 1 & 0 & 0 & \cdots & 0 & 0 & 0 & \cdots & 0 & 0 & 0 & \cdots & 0 \\
& & & & & & & & & & & & & & & \\
\star & \star & \cdots & \star & 0 & 0 & \cdots & 0 & 1 & 0 & \cdots & 0 & 0 & 0 & \cdots & 0 \\
\star & \star & \cdots & \star & \star & \star & \cdots & \star & 0 & 1 & \cdots & 0 & 0 & 0 & \cdots & 0 \\
\vdots & \vdots & \ddots & \vdots & \vdots & \vdots & & \vdots & \vdots & \vdots & \ddots & \vdots & \vdots & \vdots & & \vdots \\
\star & \star & \cdots & \star & \star & \star & \cdots & \star & 0 & 0 & \cdots & 1 & 0 & 0 & \cdots & 0
\end{array}\right) .
$$

Wc may now rcplace the stars in the last $n-m$ columns of $M_{3}$ by zeros by subtracting suitable multiples of the first $m$ columns of $M_{A}$. This may be effected through multiplying $M$ on the right with a symplectic matrix of the form

$$
\left(\begin{array}{ll}
E & 0 \\
S & E
\end{array}\right)
$$

where $S$ is a symmetric $n \times n$ matrix. Since $S$ must be symmetric, this will, at the same time alter the starred entries in the first $m$ columns of $M_{3}$, but nothing clse.

Consider next the possibility that the procedure of Step 4 has produced a series of 1's on the main diagonal, followed by a 0 . The situration is illustrated by the matrix

$$
\left(\begin{array}{cccccccccccccccc}
1 & 0 & \cdots & 0 & 0 & 0 & \cdots & 0 & 0 & 0 & \cdots & 0 & 0 & 0 & \cdots & 0 \\
0 & 1 & \cdots & 0 & 0 & 0 & \cdots & 0 & 0 & 0 & \cdots & 0 & 0 & 0 & \cdots & 0 \\
\vdots & \vdots & \ddots & \vdots & \vdots & & & \vdots & \vdots & \vdots & \ddots & \vdots & \vdots & & & \vdots \\
0 & 0 & \cdots & 1 & 0 & 0 & \cdots & 0 & 0 & 0 & \cdots & 0 & 0 & 0 & \cdots & 0 \\
& & & & & & & & & & & & & & & \\
\star & \star & \cdots & \star & 1 & 0 & \cdots & 0 & d_{1} & 0 & \cdots & 0 & 0 & 0 & \cdots & 0 \\
\star & \star & \cdots & \star & \star & 0 & \cdots & 0 & 0 & 1 & \cdots & 0 & 0 & 0 & \cdots & 0 \\
\vdots & \vdots & \ddots & \vdots & \vdots & & & \vdots & \vdots & \vdots & \ddots & \vdots & \vdots & & & \vdots \\
\star & \star & \cdots & \star & \star & \star & \cdots & \star & 0 & 0 & \cdots & 1 & 0 & 0 & \cdots & 0
\end{array}\right) .
$$

As in the previous case, the starred entries below the second row of $M_{3}$ may be replaced by zeros by subtracting suitable multiples of the columns of $M_{1}$. Note that this argument could also be carried through if the entry $1 \mathrm{in} M_{3}$ and the entry $d_{1}$ in $M_{A}$ were identity and diagonal matrices, respectively. 
The last possibility to be considered is that Step 4 leads to a matrix exemplified by the form

$$
\left(\begin{array}{cccccccccccc}
1 & 0 & \cdots & 0 & 0 & 0 & 0 & 0 & \cdots & 0 & 0 & 0 \\
0 & 1 & \cdots & 0 & 0 & 0 & 0 & 0 & \cdots & 0 & 0 & 0 \\
\vdots & \vdots & \ddots & \vdots & \vdots & \vdots & \vdots & \vdots & \ddots & \vdots & \vdots & \vdots \\
0 & 0 & \cdots & 1 & 0 & 0 & 0 & 0 & \cdots & 0 & 0 & 0 \\
& & & & & & & & & & & \\
\star & \star & \cdots & \star & 1 & 0 & d_{1} & 0 & \cdots & 0 & 0 & 0 \\
\star & \star & \cdots & \star & 0 & 1 & 0 & d_{2} & \cdots & 0 & 0 & 0 \\
\vdots & \vdots & \ddots & \vdots & \vdots & \vdots & \vdots & \vdots & \ddots & \vdots & \vdots & \vdots \\
\star & \star & \cdots & \star & \star & \star & 0 & 0 & \cdots & d_{m} & 0 & 0
\end{array}\right),
$$

where all but four entries of $M_{3}$ are starred. By the assumption of Step $1, d_{j}=1$ for $j \geq 3$, and hence the starred entries in the last two columns of $M_{3}$ may be replaced by zeros as beforc.

We have thus arrived at a matrix on the block form

$$
\left(\begin{array}{llll}
E & 0 & 0 & 0 \\
Y & X & \Delta & 0
\end{array}\right)
$$

where $X$ is on the forr described in the formulation of the theorem and $Y$ is an unknown $m \times m$ matrix. It may be replaced by a zero matrix by multiplication on the left with the matrix

$$
\left(\begin{array}{cc}
E & 0 \\
-Y & E
\end{array}\right)
$$

\section{References}

1. Al,3ers, A drian A., A Note on the Poincaré Theorem on Impure Ricmann Matrices, Ann. of Math. 36 (1935), 151-156.

2. BrLokolos, E. D., ET Al., Algebraic-geometric principles of superposition of finite-zone solutions of intcgrable non-linear equations, Russian Math. Surveys 41 (1986), 1-49.

3. ConforTo, F., "Abelsche Funktionen und algebraische Geometrie," Springer, 1956.

4. KoWAlfvskY, S., Über die Reduction einer bestimmten Klasse Abel'scher Integrale 3ten Ranges auf elliptische Integrale, Acta Math. 4 (1884), 393- -414. 
5. Poincarḱ, H., Sur les fonctions abélienncs, Anter. J. Moth 8 (1886), 239- -342.

6. Poincarí, H., Sur la réduction des intégrales abéliennes, Bull. Soc. Math. France 12 (1884), 124-143.

7. Sminnov, A. O., A Matrix Analogue of Appell's Theorem and Reductions of Multidimensional Riemann Theta-Functions, Math. USSR Sbornik 61 (1988), 379-388.

Division of Mathematical Science

The Vorwegian Institute of Technology

>-703 Trondheim

NORWNY

Rebut ol 12 de Març de 1991 